\title{
On the numerical solution to a parabolic-elliptic system with chemotactic and periodic terms using Generalized Finite Differences
}

\author{
J.J. Benito ${ }^{\mathrm{a}, 1}$, A. García ${ }^{\mathrm{a}}$, L. Gavete ${ }^{\mathrm{b}}$, M. Negreanu ${ }^{\mathrm{c}}$, F. Ureña $\mathrm{a}^{\mathrm{a}, *}$, A.M. \\ $\operatorname{Vargas}^{\mathrm{c}}$ \\ ${ }^{a}$ UNED, ETSII, Madrid, Spain \\ ${ }^{b}$ UPM, ETSIM, Madrid, Spain \\ ${ }^{c}$ Departamento de Análisis Matemático y Matemática Aplicada, UCM, Madrid, Spain
}

\begin{abstract}
In the present paper we propose the Generalized Finite Difference Method (GFDM) for numerical solution of a cross-diffusion system with chemotactic terms.

We derive the discretization of the system using a GFD scheme in order to prove and illustrate that the uniform stability behavior/ convergence of the continuous model is also preserved for the discrete model. We prove the convergence of the explicit method and give the conditions of convergence. Extensive numerical experiments are presented to illustrate the accuracy, efficiency and robustness of the GFDM.
\end{abstract}

Keywords: Chemotaxis models, Parabolic-elliptic systems, Generalized finite difference method

\section{Chemotaxis: Mathematical formulation}

Chemotaxis is the phenomenon in which cells, bacteria, and other singlecell or multicellular organisms (such as bacterias, cells of the immune system, cells of the endothelium etc.) direct their movements according to certain

\footnotetext{
*Corresponding author

Email addresses: jbenito@ind.uned.es (J.J. Benito), angelochurri@gmail.com (A. García), lu.gavete@upm.es (L. Gavete), negreanu@mat.ucm.es (M. Negreanu), furenaprieto@gmail.com (F. Ureña), antonvar@ucm.es (A.M. Vargas)
} 
chemicals (chemoattractants) in their environment. The individuals of the biological species " $U$ " are able to recognize the chemical signal " $V$ ", to measure its concentration and to move towards the higher concentrations of the substance (positive taxis) or away from it (negative taxis).

The mathematical models of chemotaxis are usually described by highly nonlinear time dependent systems of partial differential equations (PDEs). Therefore, accurate and efficient numerical methods are very important for the validation and analysis of these systems. Furthermore, a common property of all existing chemotaxis systems is their ability to model a concentration phenomenon that mathematically results in solutions rapidly growing in small neighborhoods of concentration points/curves. The solutions may blow up or may exhibit a very singular, spiky behavior. This blow-up represents a mathematical description of a cell concentration phenomenon that occurs in real biological systems. In either case, capturing such solutions numerically is a challenging problem. Mathematical models with chemotactic terms have been applied to model Angiogenesis, a key process in Tumor Growth [1], whereby endothelial cells move towards the tumor following a chemical gradient, creating new blood vessels and providing extra supply to the tumor. The mathematical model we present in this article includes a space dependence of the chemical secretion denoted by $f$, although it is not the purpose of this article to study the controllability of angiogenesis systems, the term $f$ can be considered as a control term, see for instance Delgado et al [5] where the authors have considered a flux therapy application of an anti-angiogenic therapy.

The mathematical models of PDEs concerning chemotaxis were first developed by Keller and Segel $[14,15]$. We consider a generalization of the minimal Keller-Segel model where the diffusion of the chemical substance is assumed to be dominant, i.e., its rate of production is negligible. Our numerical study is addressed to the parabolic-elliptic system

$$
\left\{\begin{array}{lr}
\frac{\partial U}{\partial t}=\Delta U-\operatorname{div}(\eta U \nabla V)+\sigma U(N(\mathbf{x}, t)-U), & \mathbf{x} \in \Omega, t>0 \\
-\Delta V+V=U, & \mathbf{x} \in \Omega, t>0 \\
U(0, \mathbf{x})=U_{0}(\mathbf{x}), & \mathbf{x} \in \Omega \\
\frac{\partial U}{\partial \nu}=\frac{\partial V}{\partial \nu}=0, & \mathbf{x} \in \partial \Omega, t>0
\end{array}\right.
$$

where " $\sigma(N(\mathbf{x}, t)-U)$ " describes the growth rate of " $U$ " and $N(\mathbf{x}, t)=$ 
$1+f(\mathbf{x}, t)$ is the carrying capacity of the system. Function " $f$ " considered in [17] and in the present paper, describing the resources of the systems, presents a periodic asymptotic behavior in the sense

$$
\lim _{t \rightarrow \infty} \sup _{\mathbf{x} \in \Omega}|f(\mathbf{x}, t)-g(t)|=0,
$$

where $g$ is independent of $\mathbf{x}$ and periodic in time. Once the threshold value $N$ is reached, the population would die because of the shortage of resources. It is natural that these resources depend on time and present some kind of periodicity caused, for instance, by the seasons of the year and consequently the population presents some seasonal behavior. The motivation of including such term can be found in [17] where it was proved the global existence of solutions and its asymptotic behavior produced by the logistic growth which counteracts the blow-up tendency produced by chemotaxis. Under suitable assumptions on the initial data and $g$, if the constant chemotactic sensitivity $\eta$ satisfies

$$
\eta<\sigma / 2
$$

the authors obtained that the solution of the system converges to a homogeneous in space and periodic in time function. Under certain assumptions, ( see Hypotheses (1.5)-(1.11) in [17]), it is expected that the solution of the system converges to a periodic function function depending on $\sigma$ and $g(t)$ which can be explicitly computed.

In our work we study the implementation of the GFDM for the KellerSegel chemotaxis model with parabolic-elliptic coupling. The explicit formulae and h-adaptative method was developed by Benito, Gavete and Ureña $[2,4,10,11,12]$. The influence of several factors as number of points, time stepping size and the choice of the weight function have been studied by the authors in $[2,9]$ and [3] ( chapter 7, 3.2. Influence of essential factors).

Recently, the GFDM has been proved to solve efficiently nonlinear PDEs. For instance, it has been used for solving nonlinear convection-diffusion equations [7], in-plane crank problems [13] and simulating water wave interactions with multiple-bottom-seated-cylinder-array structures [8], all of them emphasising the irregularity of the domains. Also, new approaches for solving highly nonlinear PDEs have been proposed as the Nonlocal Operator Method, which uses the weak form (also called energy form). Authors obtained very recently the fundamentals of the method in [19] and numerical solutions to the Electromagnetic Waveguide problem in [18].

Three particular cases are studied in this paper. Firstly, we consider the case 
$\sigma=0$, that is to say, there is no logistic source. It is well-known that the absence of such term may end up in the blow-up of solutions (i.e., solutions become unbounded at finite time). More precisely, in [16], the author proved that the solution of system (1) (when $\sigma=0$ ) blows up in finite time under the condition

$$
\int_{\Omega} U_{0}(\mathbf{x}) d \mathbf{x}>\frac{8 \pi}{\eta} .
$$

Secondly, for $0<\eta<\sigma$, we consider $f=0$, which is extensively studied in the literature. For instance, in [20] the authors proved that all solutions of the non-stationary system approach the steady state $(1,1)$ for large times. Finally, we consider a function $f(\mathbf{x}, t)$ fulfilling the assumptions made in [17] with the periodic asymptotic behavior stated in (2). We present numerical examples of all three cases in Section 3, for both regular and irregular domains.

The paper is organized as follows: in Section 2 we provide some explicit formulae using the Generalized Finite-Difference method for accurate and efficient approximations of solutions to chemotaxis models (1). Also, we study the convergence of the GFD explicit scheme. This result is enclosed in Theorem 2.1, which is the main result of the paper and where we state under which condition for the time step, $\Delta t$, the GFD explicit scheme is convergent. In Section 3, extensive numerical experiments (convergence studies in space and in time, long-time simulations, etc.) are presented to illustrate the accuracy, efficiency and robustness of the developed numerical algorithms. We finally present some conclusions.

\section{GDF explicit scheme}

In order to derive the Generalized finite difference scheme we use the following approximation of the time derivative

$$
\partial_{t} u\left(x_{0}, y_{0}, n \Delta t\right)=\frac{u_{0}^{n+1}-u_{0}^{n}}{\Delta t}+\mathcal{O}(\Delta t),
$$

and the spatial derivatives in vectorial form as obtained in $[22,11,6,21]$, that is,

$$
\boldsymbol{D}_{\mathbf{5}} u\left(\mathbf{x}_{0}, n \Delta t\right)=-\varpi_{\mathbf{0}} u_{0}^{n}+\sum_{i=1}^{s} \varpi_{\boldsymbol{i}} u_{i}^{n}+\mathcal{O}\left(h_{i}^{2}, k_{i}^{2}\right)
$$


where $\varpi_{0}$ and $\varpi_{i}$ stand for

$$
\begin{gathered}
\varpi_{0}=\left\{\varpi_{01}, \varpi_{02}, \varpi_{03}, \varpi_{04}, \varpi_{05}\right\}^{T}, \\
\varpi_{\boldsymbol{i}}=\left\{\varpi_{i 1}, \varpi_{i 2}, \varpi_{i 3}, \varpi_{i 4}, \varpi_{i 5}\right\}^{T},
\end{gathered}
$$

fulfilling

$$
\varpi_{0}=\sum_{i=1}^{s} \varpi_{i}
$$

For the sake of simplicity, let us put

$$
\varpi_{00}=\varpi_{03}+\varpi_{04} ; \quad \varpi_{i 0}=\varpi_{i 3}+\varpi_{i 4} .
$$

In order to deal with the homogeneous Neumann boundary conditions,

$$
\frac{\partial U}{\partial \nu}=\frac{\partial V}{\partial \nu}=0
$$

we use the central difference, which is of second order, if the domain $\Omega$ has regular boundary (in the sense of the distribution of nodes) and GFD formulae in other case.

We derive the discretization of the first equation. The existence and uniqueness of solutions of system (1) is proved for the case $\Omega \subset \mathbb{R}^{p}, p \in \mathbb{N}$, for system (1) though we shall consider the case $p=2$. Then, we write the first equation as

$$
\begin{aligned}
& \frac{\partial U}{\partial t}=\Delta U-\eta \nabla U \cdot \nabla V-\eta U(V-U)+\sigma U(1+f(\mathbf{x}, t)-U)= \\
& =\frac{\partial^{2} U}{\partial x^{2}}+\frac{\partial^{2} U}{\partial y^{2}}-\eta \frac{\partial U}{\partial x} \frac{\partial V}{\partial x}-\eta \frac{\partial U}{\partial y} \frac{\partial V}{\partial y}+U^{2}(\eta-\sigma)-\eta U V+\sigma U+\sigma U f(\mathbf{x}, t) .
\end{aligned}
$$

Remark 2.1. In the $3 D$ case the GFD explicit formulae reads for the spatial derivatives as

$$
\boldsymbol{D}_{\mathbf{9}} u\left(\boldsymbol{x}_{0}, n \Delta t\right)=-\varpi_{\mathbf{0}} u_{0}^{n}+\sum_{i=1}^{s} \varpi_{\boldsymbol{i}} u_{i}^{n}+\mathcal{O}\left(h_{i}^{2}, k_{i}^{2}, l_{i}^{2}\right),
$$

where $\varpi_{0}$ and $\varpi_{\boldsymbol{i}}$ now stand for

$$
\begin{gathered}
\varpi_{0}=\left\{\varpi_{01}, \varpi_{02}, \varpi_{03}, \varpi_{04}, \varpi_{05}, \varpi_{06}, \varpi_{07}, \varpi_{08}, \varpi_{09}\right\}^{T}, \\
\varpi_{i}=\left\{\varpi_{i 1}, \varpi_{i 2}, \varpi_{i 3}, \varpi_{i 4}, \varpi_{i 5}, \varpi_{i 6}, \varpi_{i 7}, \varpi_{i 8}, \varpi_{i 9}\right\}^{T},
\end{gathered}
$$

again with

$$
\varpi_{0}=\sum_{i=1}^{s} \varpi_{i}
$$


The conditional convergence of the GFD explicit scheme in 2D is addressed in the following result.

Theorem 2.1. Let $U, V \in C_{l o c}^{2,1}(\bar{\Omega} \times[0, \infty))$ the solution to the system (1). The GFD explicit scheme

$$
\left\{\begin{aligned}
u_{0}^{n+1}= & u_{0}^{n}+\Delta t\left[-\left(\varpi_{03}+\varpi_{04}\right) u_{0}^{n}+\sum_{i=1}^{s}\left(\varpi_{i 3}+\varpi_{i 4}\right) u_{i}^{n}\right]- \\
& -\eta \Delta t\left(-\varpi_{01} u_{0}^{n}+\sum_{i=1}^{s}+\varpi_{i 1} u_{i}^{n}\right)\left(-\varpi_{01} v_{0}^{n}+\sum_{i=1}^{s}+\varpi_{i 1} v_{i}^{n}\right)- \\
& -\eta \Delta t\left(-\varpi_{02} u_{0}^{n}+\sum_{i=1}^{s}+\varpi_{i 2} u_{i}^{n}\right)\left(-\varpi_{02} v_{0}^{n}+\sum_{i=1}^{s}+\varpi_{i 2} v_{i}^{n}\right)+ \\
& +\Delta t\left[\left(u_{0}^{n}\right)^{2}(\eta-\sigma)-\eta u_{0}^{n} v_{0}^{n}+\sigma u_{0}^{n}\right]+\mathcal{O}\left(\Delta t, h_{i}^{2}, k_{i}^{2}\right) \\
& v_{0}^{n}-\left[-\left(\varpi_{03}+\varpi_{04}\right) v_{0}^{n}+\sum_{i=1}^{s}\left(\varpi_{i 3}+\varpi_{i 4}\right) v_{i}^{n}\right]=u_{0}^{n}+\mathcal{O}\left(\Delta t, h_{i}^{2}, k_{i}^{2}\right)
\end{aligned}\right.
$$

is convergent under the condition

$$
0<\Delta t<\frac{2+\left|\varpi_{00}\right|+\sum_{i=1}^{s}\left|\varpi_{i 0}\right|}{\left(1+\left|\varpi_{00}\right|+\sum_{i=1}^{s}\left|\varpi_{i 0}\right|\right)\left(A_{2}+A_{3}\right)+B_{1}},
$$

where

$$
\begin{gathered}
A_{2}:=\sum_{i=1}^{s}\left|\varpi_{i 0}\right|+\left|\eta \varpi_{01} v_{0}^{n}\right| \sum_{i=1}^{s}\left|\varpi_{i 1}\right|+\left|\eta \varpi_{02} v_{0}^{n}\right| \sum_{i=1}^{s}\left|\varpi_{i 2}\right| \\
+\left|\eta \sum_{i=1}^{s} \varpi_{i 1} V_{i}^{n}\right| \sum_{i=1}^{s}\left|\varpi_{i 1}\right|+\left|\eta \sum_{i=1}^{s} \varpi_{i 2} V_{i}^{n}\right| \sum_{i=1}^{s}\left|\varpi_{i 2}\right|, \\
A_{3}:=\varpi_{00}+\eta\left(\varpi_{01}\right)^{2} V_{0}^{n}+\eta\left(\varpi_{02}\right)^{2} V_{0}^{n}-\eta\left(\varpi_{01} \sum_{i=1}^{s} \varpi_{i 1} V_{i}^{n}+\varpi_{02} \sum_{i=1}^{n} \varpi_{i 2} V_{i}^{n}\right) \\
-(\eta-\sigma)\left(u_{0}^{n}+U_{0}^{n}\right)+\eta V_{0}^{n}-\sigma\left(1+f\left(x_{0}, y_{0}, n \Delta t\right)\right),
\end{gathered}
$$


and

$$
\begin{aligned}
& B_{1}:=\left|\eta\left[\left(\varpi_{01}\right)^{2}-\left(\varpi_{02}\right)^{2}\right] u_{0}^{n}+\eta \varpi_{01} \sum_{i=1}^{s} \varpi_{i 1} u_{i}^{n}+\eta \varpi_{02} \sum_{i=1}^{s} \varpi_{i 2} u_{0}^{n}-\eta u_{0}^{n}\right| \\
& +\left|\eta \varpi_{01} U_{0}^{n}\right| \sum_{i=1}^{s}\left|\varpi_{i 1}\right|+\left|\eta \varpi_{02} U_{0}^{n}\right| \sum_{i=1}^{s}\left|\varpi_{i 2}\right|+\eta\left|\sum_{i=1}^{s} \varpi_{i 1} U_{i}^{n}\right| \sum_{i=1}^{s}\left|\varpi_{i 1}\right|+ \\
& +\eta\left|\sum_{i=1}^{s} \varpi_{i 2} U_{i}^{n}\right| \sum_{i=1}^{s}\left|\varpi_{i 2}\right| .
\end{aligned}
$$

Proof. We proceed in the following manner: let us take the difference between GFD scheme (3) and the expression for the exact solution. The time derivative is approximated by

$$
\frac{\partial u\left(x_{0}, y_{0}, n \Delta t\right)}{\partial t}=\frac{u_{0}^{n+1}-u_{0}^{n}}{\Delta t}+O(\Delta t)
$$

From the second equation of (1) we obtain

$$
u=v-\Delta v \Rightarrow \tilde{u}_{0}=\tilde{v}_{0}+\varpi_{00} \tilde{v}_{0}^{n}-\sum_{i=1}^{s} \varpi_{i 0} \tilde{v}_{i}^{n},
$$

where we use the notation, also from now on, $\tilde{u}_{j}^{n}=u_{j}^{n}-U_{j}^{n} ; \tilde{v}_{j}^{n}=v_{j}^{n}-V_{j}^{n}$ $\left(u_{j}^{n}\right.$ stands for the approximate solution at time $n \Delta t$ and point $j$ and $U_{j}^{n}$ for the exact solution). We arrive then to the following

$$
\begin{aligned}
\tilde{u}_{0}^{n+1}-\tilde{u}_{0}^{n}=\Delta t\left(-\varpi_{00} \tilde{u}_{0}^{n}+\sum_{i=1}^{s} \varpi_{i 0} \tilde{u}_{i}^{n}\right)- \\
-\eta \Delta t\left[\left(-\varpi_{01} u_{0}^{n}+\sum_{i=1}^{s} \varpi_{i 1} u_{i}^{n}\right)\left(-\varpi_{01} v_{0}^{n}+\sum_{i=1}^{s} \varpi_{i 1} v_{i}^{n}\right)\right. \\
\left.-\left(-\varpi_{01} U_{0}^{n}+\sum_{i=1}^{s} \varpi_{i 1} U_{i}^{n}\right)\left(-\varpi_{01} V_{0}^{n}+\sum_{i=1}^{s} \varpi_{i 1} V_{i}^{n}\right)\right]- \\
-\eta \Delta t\left[\left(-\varpi_{02} u_{0}^{n}+\sum_{i=1}^{s} \varpi_{i 2} u_{i}^{n}\right)\left(-\varpi_{02} v_{0}^{n}+\sum_{i=1}^{s} \varpi_{i 2} v_{i}^{n}\right)\right. \\
\left.-\left(-\varpi_{02} U_{0}^{n}+\sum_{i=1}^{s} \varpi_{i 2} U_{i}^{n}\right)\left(-\varpi_{02} V_{0}^{n}+\sum_{i=1}^{s} \varpi_{i 2} V_{i}^{n}\right)\right]+ \\
+\Delta t(\eta-\sigma)\left[\left(u_{0}^{n}\right)^{2}-\left(U_{0}^{n}\right)^{2}\right]-\Delta t \eta\left[u_{0}^{n} v_{0}^{n}-U_{0}^{n} V_{0}^{n}\right]+ \\
+\Delta t \sigma \tilde{u}_{0}^{n}\left[1+f\left(x_{0}, y_{0}, n \Delta t\right)\right]+\mathcal{O}\left(\Delta t\left(\Delta t, h_{i}^{2}, k_{i}^{2}\right)\right) .
\end{aligned}
$$


Now, we put:

$$
\begin{gathered}
\left(u_{0}^{n}\right)^{2}-\left(U_{0}^{n}\right)^{2}=\tilde{u}_{0}^{n}\left(u_{0}^{n}+U_{0}^{n}\right), \\
u_{0}^{n} v_{0}^{n}-U_{0}^{n} V_{0}^{n}=u_{0}^{n} v_{0}^{n}-u_{0}^{n} V_{0}^{n}+u_{0}^{n} V_{0}^{n}-U_{0}^{n} V_{0}^{n}=u_{0}^{n} \tilde{v}_{0}^{n}+\tilde{u}_{0}^{n} V_{0}^{n},
\end{gathered}
$$

and

$$
\begin{aligned}
& \left(\varpi_{01}\right)^{2} u_{0}^{n} v_{0}^{n}-\varpi_{01} u_{0}^{n} \sum_{i=1}^{s} \varpi_{i 1} v_{i}^{n}-\varpi_{01} v_{0}^{n} \sum_{i=1}^{s} \varpi_{i 1} u_{i}^{n}+ \\
& +\left(\sum_{i=1}^{s} \varpi_{i 1} v_{i}^{n}\right)\left(\sum_{i=1}^{s} \varpi_{i 1} u_{i}^{n}\right)-\left[\left(\varpi_{01}\right)^{2} U_{0}^{n} V_{0}^{n}-\varpi_{01} U_{0}^{n} \sum_{i=1}^{s} \varpi_{i 1} V_{i}^{n}-\right. \\
& \left.-\varpi_{01} V_{0}^{n} \sum_{i=1}^{s} \varpi_{i 1} U_{i}^{n}+\left(\sum_{i=1}^{s} \varpi_{i 1} V_{i}^{n}\right)\left(\sum_{i=1}^{s} \varpi_{i 1} U_{i}^{n}\right)\right] \\
& =\left(\varpi_{01}\right)^{2}\left[u_{0}^{n} v_{0}^{n}-U_{0}^{n} V_{0}^{n}\right]-\varpi_{01}\left[u_{0}^{n} \sum_{i=1}^{s} \varpi_{i 1} v_{i}^{n}-U_{0}^{n} \sum_{i=1}^{s} \varpi_{i 1} V_{i}^{n}\right]- \\
& -\varpi_{01}\left[v_{0}^{n} \sum_{i=1}^{s} \varpi_{i 1} u_{i}^{n}-V_{0}^{n} \sum_{i=1}^{s} \varpi_{i 1} U_{i}^{n}\right] \\
& +\left[\left(\sum_{i=1}^{s} \varpi_{i 1} v_{i}^{n}\right)\left(\sum_{i=1}^{s} \varpi_{i 1} u_{i}^{n}\right)-\left(\sum_{i=1}^{s} \varpi_{i 1} V_{i}^{n}\right)\left(\sum_{i=1}^{s} \varpi_{i 1} U_{i}^{n}\right)\right] .
\end{aligned}
$$

We can rewrite (8) as

$$
\begin{aligned}
(8)= & \left(\varpi_{01}\right)^{2}\left[u_{0}^{n} \tilde{v}_{0}^{n}+\tilde{u}_{0}^{n} V_{0}^{n}\right]-\varpi_{01}\left(\tilde{u}_{0}^{n} \sum_{i=1}^{s} \varpi_{i 1} V_{i}^{n}+U_{0}^{n} \sum_{i=1}^{s} \varpi_{i 1} \tilde{v}_{i}^{n}\right) \\
& -\varpi_{01}\left(\tilde{v}_{0}^{n} \sum_{i=1}^{s} \varpi_{i 1} u_{i}^{n}+V_{0}^{n} \sum_{i=1}^{s} \varpi_{i 1} \tilde{u}_{i}^{n}\right)+ \\
& +\left(\sum_{i=1}^{s} \varpi_{i 1} \tilde{u}_{i}^{n}\right)\left(\sum_{i=1}^{s} \varpi_{i 1} V_{i}^{n}\right)+\left(\sum_{i=1}^{s} \varpi_{i 1} U_{i}^{n}\right)\left(\sum_{i=1}^{s} \varpi_{i 1} \tilde{v}_{i}^{n}\right) .
\end{aligned}
$$


Now, substituting (6), (7) and (9) all together in (5)

$$
\begin{aligned}
\tilde{u}_{0}^{n+1} & -\tilde{u}_{0}^{n}=\Delta t\left(-\varpi_{00} \tilde{u}_{0}^{n}+\sum_{i=1}^{s} \varpi_{i 0} \tilde{u}_{i}^{n}\right)- \\
& -\Delta t \eta\left(\varpi_{01}\right)^{2}\left[u_{0}^{n} \tilde{v}_{0}^{n}+\tilde{u}_{0}^{n} V_{0}^{n}\right]-\Delta t \eta\left(\varpi_{02}\right)^{2}\left[u_{0}^{n} \tilde{v}_{0}^{n}+\tilde{u}_{0}^{n} V_{0}^{n}\right] \\
& +\Delta t \eta \varpi_{01}\left(\tilde{u}_{0}^{n} \sum_{i=1}^{s} \varpi_{i 1} V_{i}^{n}+U_{0}^{n} \sum_{i=1}^{s} \varpi_{i 1} \tilde{v}_{i}^{n}\right)+ \\
& +\Delta t \eta \varpi_{02}\left(\tilde{u}_{0}^{n} \sum_{i=1}^{s} \varpi_{i 2} V_{i}^{n}+U_{0}^{n} \sum_{i=1}^{s} \varpi_{i 2} \tilde{v}_{i}^{n}\right) \\
& +\Delta t \eta \varpi_{01}\left(\tilde{v}_{0}^{n} \sum_{i=1}^{s} \varpi_{i 1} u_{i}^{n}+V_{0}^{n} \sum_{i=1}^{s} \varpi_{i 1} \tilde{u}_{i}^{n}\right)+ \\
& +\Delta t \eta \varpi_{02}\left(\tilde{v}_{0}^{n} \sum_{i=1}^{s} \varpi_{i 2} u_{i}^{n}+V_{0}^{n} \sum_{i=1}^{s} \varpi_{i 2} \tilde{u}_{i}^{n}\right) \\
& -\Delta t \eta\left(\sum_{i=1}^{s} \varpi_{i 1} \tilde{u}_{i}^{n}\right)\left(\sum_{i=1}^{s} \varpi_{i 1} V_{i}^{n}\right)-\Delta t \eta\left(\sum_{i=1}^{s} \varpi_{i 2} \tilde{u}_{i}^{n}\right)\left(\sum_{i=1}^{s} \varpi_{i 2} V_{i}^{n}\right) \\
& -\Delta t \eta\left(\sum_{i=1}^{s} \varpi_{i 1} U_{i}^{n}\right)\left(\sum_{i=1}^{s} \varpi_{i 1} \tilde{v}_{i}^{n}\right)-\Delta t \eta\left(\sum_{i=1}^{s} \varpi_{i 2} U_{i}^{n}\right)\left(\sum_{i=1}^{s} \varpi_{i 2} \tilde{v}_{i}^{n}\right) \\
& +\Delta t \tilde{u}_{0}^{n}(\eta-\sigma)\left(u_{0}^{n}+U_{0}^{n}\right)-\Delta t \eta\left(u_{0}^{n} \tilde{v}_{0}^{n}+\tilde{u}_{0}^{n} V_{0}^{n}\right)+ \\
+ & \Delta t \sigma \tilde{u}_{0}^{n}\left[1+f\left(x_{0}, y_{0}, n \Delta t\right)\right]+\mathcal{O}\left(\Delta t\left(\Delta t, h_{i}^{2}, k_{i}^{2}\right)\right) .
\end{aligned}
$$


Now, it yields

$$
\begin{aligned}
\tilde{u}_{0}^{n+1} & =\tilde{u}_{0}^{n}\left[1-\varpi_{00} \Delta t-\Delta t \eta\left(\varpi_{01}\right)^{2} V_{0}^{n}-\Delta t \eta\left(\varpi_{02}\right)^{2} V_{0}^{n}+\right. \\
& +\Delta t \eta\left(\varpi_{01} \sum_{i=1}^{s} \varpi_{i 1} V_{i}^{n}+\varpi_{02} \sum_{i=1}^{n} \varpi_{i 2} V_{i}^{n}\right) \\
& +\Delta t(\eta-\sigma)\left(u_{0}^{n}+U_{0}^{n}\right)-\Delta t \eta V_{0}^{n}+\Delta t \sigma\left[1+f\left(x_{0}, y_{0}, n \Delta t\right)\right]+ \\
& +\Delta t\left[\sum_{i=1}^{s} \tilde{u}_{i}^{n}+\eta \varpi_{01} V_{0}^{n} \sum_{i=1}^{s} \varpi_{i 1} \tilde{u}_{i}^{n}+\eta \varpi_{02} V_{0}^{n} \sum_{i=1}^{s} \varpi_{i 2} \tilde{u}_{i}^{n}\right. \\
& -\left(\eta \sum_{i=1}^{s} \varpi_{i 1} V_{i}^{n}\right) \sum_{i=1}^{s} \varpi_{i 1} \tilde{u}_{i}^{n}-\left(\eta \sum_{i=1}^{s} \varpi_{i 2} V_{i}^{n} \sum_{i=1}^{s} \varpi_{i 2} \tilde{u}_{i}^{n}\right]+ \\
& +\Delta t \tilde{v}_{0}^{n}\left[\left(-\eta\left(\varpi_{01}\right)^{2}-\eta\left(\varpi_{02}\right)^{2}\right) u_{0}^{n}+\eta \varpi_{01} \sum_{i=1}^{s} \varpi_{i 1} u_{i}^{n}+\right. \\
& \left.+\eta \varpi_{02} \sum_{i=1}^{s} \varpi_{i 2} u_{i}^{n}-\eta u_{0}^{n}\right]+\Delta t\left[\eta \varpi_{01} U_{0}^{n} \sum_{i=1}^{s} \varpi_{i 1} \tilde{v}_{i}^{n}\right. \\
& +\eta \varpi_{02} U_{0}^{n} \sum_{i=1}^{s} \varpi_{i 2} \tilde{v}_{i}^{n}-\eta\left(\sum_{i=1}^{s} \varpi_{i 1} U_{i}^{n}\right) \sum_{i=1}^{s} \varpi_{i 1} \tilde{v}_{i}^{n} \\
& -\eta\left(\sum_{i=1}^{s} \varpi_{i 2} U_{i}^{n}\right) \sum_{i=1}^{s} \varpi_{i 2} \tilde{v}_{i}^{n}+\mathcal{O}\left(\Delta t\left(\Delta t, h_{i}^{2}, k_{i}^{2}\right)\right) .
\end{aligned}
$$

Let us define $\tilde{u}^{n}=\max _{i \in\{0, \ldots, s\}}\left|\tilde{u}_{i}^{n}\right|$ and $\tilde{v}^{n}=\max _{i \in\{0, \ldots, s\}}\left|\tilde{v}_{i}^{n}\right|$. We rewrite 
(10) as follows

$$
\begin{aligned}
\tilde{u}^{n+1} & \leq \tilde{u}^{n}\left[\mid 1-\Delta t\left(\varpi_{00}+\eta\left(\varpi_{01}\right)^{2} V_{0}^{n}+\eta\left(\varpi_{02}\right)^{2} V_{0}^{n}-\right.\right. \\
& \eta\left(\varpi_{01} \sum_{i=1}^{s} \varpi_{i 1} V_{i}^{n}+\varpi_{02} \sum_{i=1}^{n} \varpi_{i 2} V_{i}^{n}\right)-(\eta-\sigma)\left(u_{0}^{n}+U_{0}^{n}\right)+\eta V_{0}^{n} \\
& \left.-\sigma\left(1+f\left(x_{0}, y_{0}, n \Delta t\right)\right)\right) \mid+\Delta t\left(\sum_{i=1}^{s}\left|\varpi_{i 0}\right|+\left|\eta \varpi_{01} V_{0}^{n}\right| \sum_{i=1}^{s}\left|\varpi_{i 1}\right|\right. \\
& +\left|\eta \varpi_{02} V_{0}^{n}\right| \sum_{i=1}^{s}\left|\varpi_{i 2}\right|+\left|\eta \sum_{i=1}^{s} \varpi_{i 1} V_{i}^{n}\right| \sum_{i=1}^{s}\left|\varpi_{i 1}\right|+ \\
& \left.\left.+\left|\eta \sum_{i=1}^{s} \varpi_{i 2} V_{i}^{n}\right| \sum_{i=1}^{s}\left|\varpi_{i 2}\right|\right)\right]+\Delta t \tilde{v}^{n}\left[\mid \eta\left[-\left(\varpi_{01}\right)^{2}-\left(\varpi_{02}\right)^{2}\right] u_{0}^{n}+\right. \\
& +\eta \varpi_{01} \sum_{i=1}^{s} \varpi_{i 1} u_{i}^{n}+\eta \varpi_{02} \sum_{i=1}^{s} \varpi_{i 2} u_{i}^{n}-\eta u_{0}^{n} \mid \\
& +\left|\eta \varpi_{01} U_{0}^{n}\right| \sum_{i=1}^{s}\left|\varpi_{i 1}\right|+\left|\eta \varpi_{02} U_{0}^{n}\right| \sum_{i=1}^{s}\left|\varpi_{i 2}\right|+ \\
& \left.+\eta\left|\sum_{i=1}^{s} \varpi_{i 1} U_{i}^{n}\right| \sum_{i=1}^{s}\left|\varpi_{i 1}\right|+\eta\left|\sum_{i=1}^{s} \varpi_{i 2} U_{i}^{n}\right| \sum_{i=1}^{s}\left|\varpi_{i 2}\right|\right]+\mathcal{O}\left(\Delta t\left(\Delta t, h_{i}^{2}, k_{i}^{2}\right)\right) .
\end{aligned}
$$

For the sake of simplicity, let us put

$$
\tilde{u}^{n+1} \leq A \tilde{u}^{n}+B \tilde{v}^{n} .
$$

Notice that since

$$
\left|\tilde{v}_{0}^{n}+\varpi_{00} \tilde{v}_{0}^{n}-\sum_{i=1}^{s} \varpi_{i 0} \tilde{v}_{i}^{n}\right|=\left|\tilde{u}_{0}^{n}\right|,
$$

we have

$$
\tilde{v}^{n}\left[\left|1+\varpi_{00}\right|+\sum_{i=1}^{s}\left|\varpi_{i 0}\right|\right] \geq \tilde{u}^{n} .
$$

Now, by combining (12) with (14) we get

$$
\tilde{u}^{n+1} \leq\left(A\left[1+\left|\varpi_{00}\right|+\sum_{i=1}^{s}\left|\varpi_{i 0}\right|\right]+B\right) \tilde{v}^{n} .
$$


The stabilibity condition follows from

$$
\alpha:=A\left[1+\left|\varpi_{00}\right|+\sum_{i=1}^{s}\left|\varpi_{i 0}\right|\right]+B<1 .
$$

Let us call $A=A_{1}+\Delta t A_{2}=\left|1-\Delta t A_{3}\right|+\Delta t A_{2}$ and $B=B_{1} \Delta t$. Hence, the following holds

$$
\left|1-\Delta t A_{3}\right|<\frac{1-B_{1} \Delta t}{1+\left|\varpi_{00}\right|+\sum_{i=1}^{s}\left|\varpi_{i 0}\right|}-\Delta t A_{2},
$$

or equivalently,

$$
-\frac{1-B_{1} \Delta t}{1+\left|\varpi_{00}\right|+\sum_{i=1}^{s}\left|\varpi_{i 0}\right|}+\Delta t A_{2}<1-\Delta t A_{3}<\frac{1-B_{1} \Delta t}{1+\left|\varpi_{00}\right|+\sum_{i=1}^{s}\left|\varpi_{i 0}\right|}-\Delta t A_{2} .
$$

Then, we have

$$
1+\frac{1-B_{1} \Delta t}{1+\left|\varpi_{00}\right|+\sum_{i=1}^{s}\left|\varpi_{i 0}\right|}-\Delta t A_{2}>\Delta t A_{3}>1-\frac{1-B_{1} \Delta t}{1+\left|\varpi_{00}\right|+\sum_{i=1}^{s}\left|\varpi_{i 0}\right|}+\Delta t A_{2} .
$$

From the LHS inequality of (19) we obtain

$$
\Delta t<\frac{2+\left|\varpi_{00}\right|+\sum_{i=1}^{s}\left|\varpi_{i 0}\right|}{\left(1+\left|\varpi_{00}\right|+\sum_{i=1}^{s}\left|\varpi_{i 0}\right|\right)\left(A_{2}+A_{3}\right)+B_{1}} .
$$

From the RHS we get

$$
\Delta t>\frac{\left|\varpi_{00}\right|+\sum_{i=1}^{s}\left|\varpi_{i 0}\right|}{\left(1+\left|\varpi_{00}\right|+\sum_{i=1}^{s}\left|\varpi_{i 0}\right|\right)\left(A_{3}-A_{2}\right)-B_{1}} .
$$

Now, since $A_{2}>A_{3}$ and $B_{1}>0$, the denominator of the RHS of (21) is negative, so we have

$$
\Delta t>0
$$

Remark 2.2. Although the aim of this work is to present a rigurous proof of the conditional convergence of the GFD explicit scheme in 2D, the same result in the three-dimensional case is achieved by introducing minor changes in the previous proof. By using the formulae given in Remark 2.1 and calling

$$
\varpi_{00}=\varpi_{04}+\varpi_{05}+\varpi_{06}, \quad \varpi_{i 0}=\varpi_{i 4}+\varpi_{i 5}+\varpi_{i 6},
$$

equations (20) and (21) are obtained for a similar definition of $A_{2}, A_{3}$ and $B_{1}$. 


\section{Numerical Results}

In this section we show the numerical results obtained by solving the system (1), using both regular and irregular clouds of points as seen in Figure 1 (441 nodes in each one) in the domain $\Omega=[0,1] \times[0,1]$. We use an 8node scheme, chosen by the distance criterion together with weight function $w=\frac{1}{d i s t^{4}}$. For all numerical examples we put $\Delta t=0.001$.
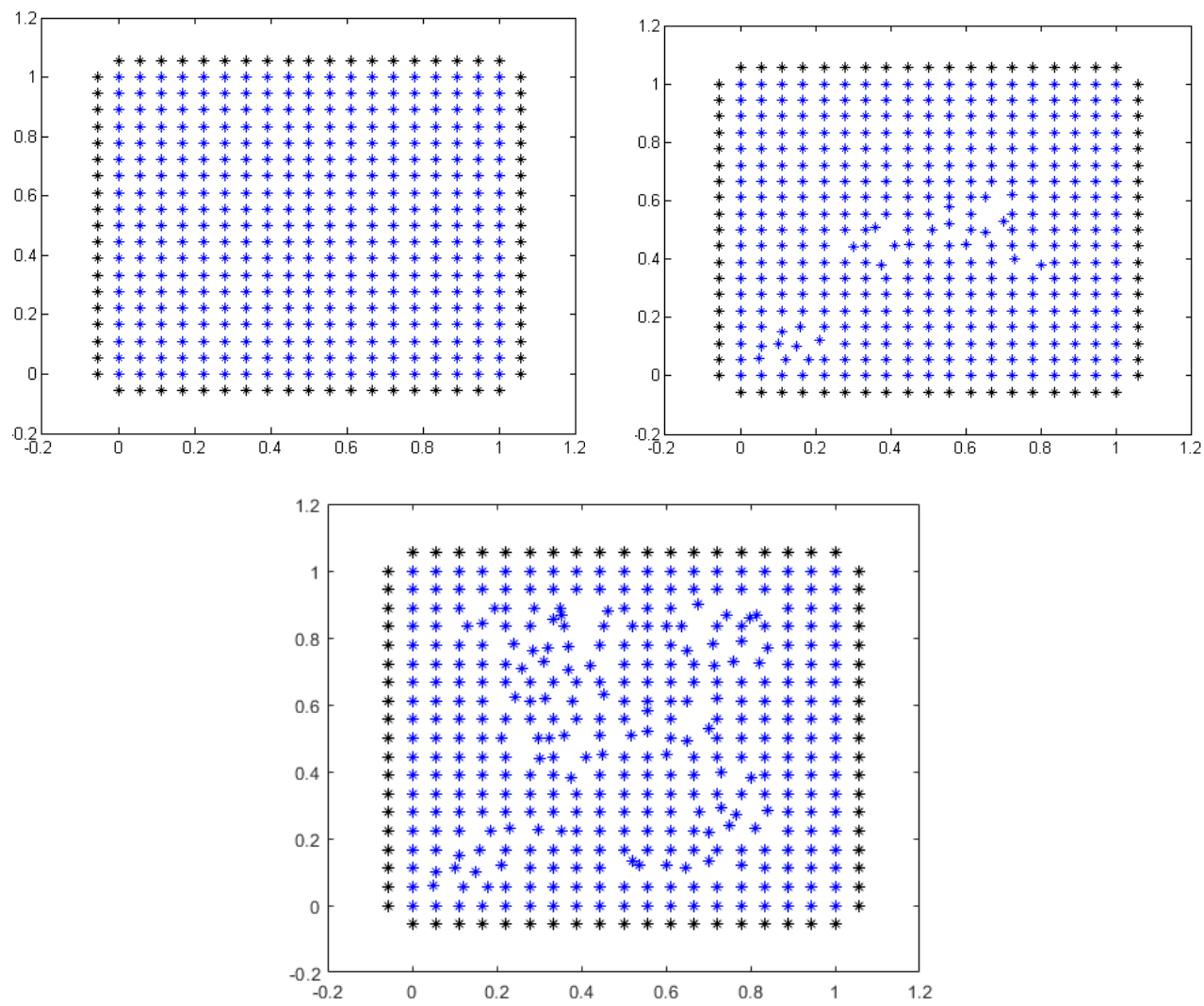

Figure 1: Regular and irregular clouds of points 


\begin{tabular}{cccccc}
\hline $\mathrm{T}(\mathrm{s})$ & 0.3 & 1 & 1.6 & 1.625 & 1.65 \\
\hline$\|u\|_{l^{\infty}(\Omega)}$ & 150.000 & 150.0041 & 449.9381 & $1.4231 \mathrm{e}+03$ & - \\
\hline$\|v\|_{l^{\infty}(\Omega)}$ & 150.000 & 150.0004 & 165.8602 & 184.2503 & - \\
\hline
\end{tabular}

Table 1: Values of $\|u\|_{l^{\infty}(\Omega)}$ and $\|v\|_{l^{\infty}(\Omega)}$ for different time values in the Example 1.

\subsection{Case 1}

In our first case we consider no source term in the $U$-equation, that is to say, we consider the minimal Keller-Segel parabolic-elliptic system:

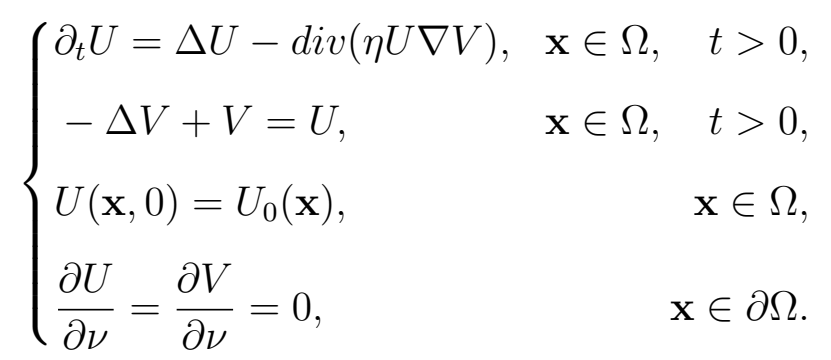

As stated, we expect to find blow up of solutions in finite time for a large enough initial data.

\subsubsection{Example 1: $\sigma=0$}

For this example we choose $\eta=0.2$ and $U_{0}(\mathbf{x})=150$, fulfilling $\int_{\Omega} U_{0}(\mathbf{x})>$ $40 \pi$. We use the irregular cloud of points of Figure 1. Table 1 show the $l^{\infty}$ norm of the solution. We obtain that the solution blows up before 1.65 seconds. We present the numerical solution (both $u$ and $v$ ) to the system in Figure 2 and obtain that, after very slow growth, solutions become unbounded at finite time.

\subsection{Case 2}

In this second case we use the GFDM to solve system (1) numerically for $f(\mathbf{x}, t)=0$. It is known that the solution to the parabolic-elliptic system has the following asymtoptic behavior:

$$
\lim _{t \rightarrow \infty}\left[\|U(\cdot, t)-1\|_{l^{\infty}(\Omega)}+\|V(\cdot, t)-1\|_{l^{\infty}(\Omega)}\right]=0,
$$

for any nonnegative continuous initial data $U_{0}(\mathbf{x})$ (see Tello and Winkler [20]). 


\subsubsection{Example 2}

In this second example we consider the following function $f(\mathbf{x}, t)=0$ and initial data $U_{0}(\mathbf{x})=3 e^{-10\left((x-0.5)^{2}+(y-0.5)^{2}\right)}$. We choose the parameters to be $\sigma=0.7$ and $\eta=0.2$. In Table 2 we present the obtained values of the maximum difference between the approximate solution, $u$, and the steady state in a regular cloud of points. Figure 3 shows the solution to the $U$ equation.

\begin{tabular}{ccccccc}
\hline $\mathrm{T}(\mathrm{s})$ & 0 & 0.03 & 0.06 & 0.1 & 1 & 6 \\
\hline$\|u-1\|_{l^{\infty}(\Omega)}$ & 2 & 0.4953 & 0.2373 & 0.1354 & 0.0616 & 0.0020 \\
\hline$\|v-1\|_{l^{\infty}(\Omega)}$ & - & 0.1247 & 0.1158 & 0.1104 & 0.0616 & 0.0020 \\
\hline
\end{tabular}

Table 2: Values of $\|u-1\|_{l^{\infty}(\Omega)}$ and $\|v-1\|_{l^{\infty}(\Omega)}$ for different time values in the Example 2 .

\subsubsection{Example 3}

Now, we consider an irregular cloud of points for the initial data $U_{0}(\mathbf{x})=$ $0.1 e^{-10\left((x-0.3)^{2}+\left((y-0.3)^{2}\right)\right.}$, the same function $f(\mathbf{x}, t)=0$ and also $\sigma=0.7$, $\eta=0.2$. Figure 3.2.1 shows the solution to the $U$-equation for different time values. Table 3 shows the values of the maximum difference between the numerical solution and the asymptotic value. Due to the small initial density

\begin{tabular}{cccccccc}
\hline $\mathrm{T}(\mathrm{s})$ & 0 & 0.03 & 0.06 & 0.3 & 1 & 6 & 10 \\
\hline$\|u-1\|_{l^{\infty}(\Omega)}$ & 1 & 0.9979 & 0.9931 & 0.9712 & 0.9497 & 0.3633 & 0.0335 \\
\hline$\|v-1\|_{l^{\infty}(\Omega)}$ & - & 0.9764 & 0.9753 & 0.9688 & 0.9498 & 0.3635 & 0.0335 \\
\hline
\end{tabular}

Table 3: Values of $\|u-1\|_{l^{\infty}(\Omega)}$ and $\|v-1\|_{l^{\infty}(\Omega)}$ for different time values for the Example 3 in the second cloud of points of Figure 1.

of population, it takes greater time to become uniform for the solution.

\subsubsection{Example 4}

We consider for this example the initial data

$$
U_{0}(\mathbf{x})=2 e^{-10\left((x-0.8)^{2}+\left((y-0.8)^{2}\right)\right.}
$$


and the parameters $\sigma=1.5$ and $\eta=0.5$ in the third cloud of points of Figure 1. Table 4 shows the $l^{\infty}$ norm of the difference between the value of the numerical solution and the expected limit at different times. Figure 5 shows the $u$-solution at such times.

\begin{tabular}{cccccccc}
\hline $\mathrm{T}(\mathrm{s})$ & 0 & 0.5 & 1 & 3 & 6 & 10 & 15 \\
\hline$\|u-1\|_{l^{\infty}(\Omega)}$ & 1 & 0.4270 & 0.2545 & 0.0167 & $1.8865 \mathrm{e}-04$ & $4.6750 \mathrm{e}-07$ & $2.5868 \mathrm{e}-10$ \\
\hline$\|v-1\|_{l^{\infty}(\Omega)}$ & - & 0.4202 & 0.2545 & 0.0167 & $1.8868 \mathrm{e}-04$ & $4.6757 \mathrm{e}-07$ & $2.5875 \mathrm{e}-10$ \\
\hline
\end{tabular}

Table 4: Values of $\|u-1\|_{l^{\infty}(\Omega)}$ and $\|v-1\|_{l^{\infty}(\Omega)}$ for different time values for the Example 4 in the third cloud of points of Figure 1.

\subsection{Case 3}

The aim of this third case is to show the asymptotic behavior of the solution $(U, V)$ of the system (1) when we consider a function $f(\mathbf{x}, t)$ fulfilling (2). In order to solve system (1) numerically we choose the function $f(\mathbf{x}, t)$ to be

$$
f(\mathbf{x}, t)=\frac{\cos t}{4+\sin t}+\frac{x-y}{1+t^{2}}, \quad(x, y) \in \Omega=[0,1] \times[0,1], t>0 .
$$

It is easily checked that this function fulfils all assumptions stated in [17]. Then, we find the $2 \pi$-periodic function

$$
g(t)=\frac{\cos t}{4+\sin t},
$$

which, gives us the $2 \pi$-periodic limit of the solution $(U, V)$,

$$
L(t)=\frac{4+\sin (t)}{4-\frac{\cos (t)}{2}+\frac{\sin (t)}{2}} .
$$

As in the previous one, we divide this case into the regular and irregular cloud of points of Figure 1, to show that in both situations, the solution to (1) inherits the periodic behavior of the function $f(\mathbf{x}, t)$ :

$$
\lim _{t \rightarrow \infty}\left[\|U(\cdot, t)-L(t)\|_{l^{\infty}(\Omega)}+\|V(\cdot, t)-L(t)\|_{l^{\infty}(\Omega)}\right]=0,
$$




\subsubsection{Example 5}

As stated, let us consider the function $f(\mathbf{x}, t)=\frac{\cos t}{4+\sin t}+\frac{x-y}{1+t^{2}}$. Assume $U_{0}(\mathbf{x})=5 e^{-10\left((x-0.6)^{2}+(y-0.6)^{2}\right)}$ as initial data and the relation of parameters $\sigma=1$ and $\eta=0.3$. Table 5 presents the values of the limit function $L(t)$ and maximum difference between this and the numerical solution.

Figure 6 shows the asymptotic solution $L$ (solid line) and the most distant values of approximation at different times. As we see, the numerical solution is also periodic and the $l^{\infty}(\Omega)$ of the difference is small for large enough times.

\begin{tabular}{ccccccc}
\hline $\mathrm{T}(\mathrm{s})$ & 1.5 & 3 & 4.5 & 6 & $1.5+2 \pi$ & $3+2 \pi$ \\
\hline$L$ & 1.1197 & 0.9070 & 0.8357 & 1.1007 & 1.1200 & 0.9075 \\
\hline$\|u-L\|_{l^{\infty}(\Omega)}$ & 0.0707 & $1.4721 \mathrm{e}-02$ & $5.1705 \mathrm{e}-03$ & $3.1418 \mathrm{e}-03$ & $1.4587 \mathrm{e}-03$ & $9.4562 \mathrm{e}-04$ \\
\hline$\|v-L\|_{l^{\infty}(\Omega)}$ & 0.0432 & 0.0079 & 0.0021 & $7.9842 \mathrm{e}-04$ & $2.6320 \mathrm{e}-04$ & $5.3863 \mathrm{e}-05$ \\
\hline
\end{tabular}

Table 5: Values of the function $L(t)$ and the differences $\|u-L\|_{l^{\infty}(\Omega)}$ and $\|v-L\|_{l^{\infty}(\Omega)}$ in the Example 5 in the regular cloud of points of Figure 1.

\subsubsection{Example 6}

For this last example we also consider the function $f(\mathbf{x}, t)$ of the previous one as well as the same relation of parameters. Let us use now the initial data

$$
U_{0}(\mathbf{x})=e^{-10\left[(x-0.7)^{2}+(y-0.7)^{2}\right]},
$$

and test the method in the second cloud of points of Figure 1. Table 6 presents the values of the limit function $L(t)$ and maximum difference between this and the numerical solution.

\begin{tabular}{ccccccc}
\hline $\mathrm{T}(\mathrm{s})$ & 1.5 & 3 & 6 & $1.5+2 \pi$ & $3+2 \pi$ & $6+2 \pi$ \\
\hline$L$ & 1.1197 & 0.9070 & 1.1007 & 1.1200 & 0.9075 & 1.1001 \\
\hline$\|u-L\|_{l^{\infty}(\Omega)}$ & 0.4401 & 0.1120 & 0.0118 & 0.0028 & 0.0012 & $6.9721 \mathrm{e}-04$ \\
\hline$\|v-L\|_{l^{\infty}(\Omega)}$ & 0.4234 & 0.1058 & 0.0098 & 0.0014 & $2.8953 \mathrm{e}-04$ & $1.0059 \mathrm{e}-04$ \\
\hline
\end{tabular}

Table 6: Values of the function $L(t)$ and the differences $\|u-L\|_{l^{\infty}(\Omega)}$ and $\|v-L\|_{l_{\infty}(\Omega)}$ in the Example 6 in the second cloud of points of Figure 1.

Note that in Figure 7, it takes longer for the approximate solution to reach the limit value $L(t)$ because the initial data is smaller than in Example 5. This means that the population's density grows slowly at initial times. Also notice that $L(t)$ is not a solution of the system but an asymptotic value for the exact solution. 


\section{Conclusions}

We have derived the discretization of the modified Keller-Segel system (1) and proved unider which condition the GFD scheme is convergent.

For Case 1, where there is no source term, we obtain numerical solutions which blow up in finite time for large enough initial data in the discrete model, in accordance with the analytical studies. For Case 2, (case $f(\mathbf{x}, t)=0$ ), we obtain asymptotic convergence to $(1,1)$ as stated for different initial data, in both regular and irregular clouds of points. No difference between these are found. Notice that the diffusion is faster when a large amount of the initial data is above the threshold value 1 . When the initial population's density is small, it takes much longer to reach this value.

For Case 3, we have obtained an approximation to the solution of the system which inherits the periodic behavior of the function $g(t)$. It is also remarkable that the elliptic equation for the chemical substance, $v$, models a fast diffusion process. Therefore the $v$-component of the solution becomes rather uniform at small times.

The Generalized Finite Difference Method solves this strongly coupled highly nonlinear parabolic-elliptic system efficiently and with high accuracy over regular and irregular domains. This means that this meshless method is an efficient tool for obtaining the numerical solution of this parabolic-elliptic chemotaxis system appearing in Biology and Medicine.

\section{Acknowledgements}

The authors acknowledge the support of the Escuela Técnica Superior de Ingenieros Industriales (UNED) of Spain, project 2019-IFC02, and of the Universidad Politécnica de Madrid (UPM) (Research groups 2019). This work is also partially support by the Project MTM2013-42907-P from MICINN (Spain).

\section{References}

[1] Anderson A.R., Chaplain M.A., Continuous and discrete mathematical models of tumor-induced angiogenesis. Bull Math Biol. 60 (5), 857-899 (1998).

[2] Benito J. J., Ureña F., Gavete L. Influence of several factors in the generalized finite difference method. Applied Mathematical Modeling; 25: 1039-1053, (2001). 
[3] Benito J. J., Ureña F., Gavete L. Leading-Edge Applied Mathematical Modelling Research. Nova Science Publishers, New York, (2008).

[4] Benito J. J., Ureña F., Gavete L. Solving parabolic and hyperbolic equations by the generalized finite difference method. Journal of Computational and Applied Mathematics ; 209: 208-233, (2007).

[5] Delgado M, Morales-Rodrigo C, Suárez A. Anti-angiogenic therapy based on the binding to receptors. Discrete and Continuous Dynamical Systems - A, 32(11), 3871-3894, (2012).

[6] Fan C. M., Huang Y.K., Li P.W., Chiu C. L. Application of the generalized finite-difference method to inverse biharmonic boundary value problems. Numerical Heat Transfer, Part B: Fundamentals, 65(2), 129$154,(2014)$.

[7] Fu Z.J., Tang Z.C., Zhao H.T., Li P.W., Rabczuk T. Numerical solutions of the coupled unsteady nonlinear convection-diffusion equations based on generalized finite difference method. The European Physical Journal Plus, 134, 272, (2019).

[8] Fu Z.J., Xie Z.Y., Zhao H.T., Ji S.Y., Tsai C.C., Li A.L. Meshless generalized finite difference method for water wave interactions with multiple-bottom-seated-cylinder-array structures. Ocean Engineering, 195: 106736 (2020).

[9] Gavete L.,Alonso B., Benito J. J., Ureña F. Application of the generalized finite difference method to improve the approximated solution of PDEs. Comput. Model. Eng. Sci.; 38: 39-58, (2009).

[10] Gavete L., Benito J. J., Ureña F. Generalized finite differences for solving $3 D$ elliptic and parabolic equations. Applied Mathematical Modelling ; 40: 955-965, (2016).

[11] Gavete L., Gavete M. L., Ureña F., Benito J. J. An Approach to Refinement of Irregular Clouds of Points Using Generalized Finite Differences. Mathematical Problems in engineering, DOI:10.1155/2015/283757. (2015). 
[12] Gavete L., Ureña F., Benito J. J., Garcia A., Ureña M., Salete E. Solving second order non-linear elliptic partial differential equations using generalized finite difference method. Journal of Computational and Applied Mathematics ; 318: 378-387, (2017).

[13] Jun L., Yanjie X., Yan G., Fan C.M. The generalized finite difference method for in-plane crack problems. Engineering Analysis with Boundary Elements, 98: 147-156( 2019).

[14] Keller EF., Segel LA. Initiation of slime mold aggregation viewed as an instability. J. Theoret. Biol. 26, 399-415, (1970).

[15] Keller EF., Segel LA. A model for chemotaxis. J. Theoret. Biol. 30, 225-234, (1971).

[16] Nagai T., Blowup of nonradial solutions to parabolic-elliptic systems modeling chemotaxis in two-dimensional domains. Journal of Inequalities and Applications 6 (1): 37-55, (2001).

[17] Negreanu M., Tello J.I., Vargas A.M., On a Parabolic-Elliptic chemotaxis system with periodic asymptotic behavior. Math Meth Appl Sci., 42 (4), 1210-1226 (2019).

[18] Rabczuk T., Ren H., Zhuang X., A Nonlocal Operator Method for Partial Differential Equations with Application to Electromagnetic Waveguide Problem. Computers, Materials and Continua, 59(1),31-55 (2019).

[19] Ren H., Zhuang X., Rabczuk T., A nonlocal operator method for solving partial differential equations. Computer Methods in Applied Mechanics and Engineering, 358, 112621, (2020).

[20] Tello JI, Winkler M. A chemotaxis system with logistic source. Communications in Partial Differential Equations 32 (6), 849-877, (2007).

[21] Ureña F., Gavete L., Garcia A., Benito J. J., Vargas A. M., Solving second order non-linear parabolic PDEs using generalized finite difference method (GFDM). Journal of Computational and Applied Mathematics; 354: 221-241, (2019).

[22] Ureña F, Gavete L, García A, Benito J.J, . Vargas A.M., Non-linear Fokker-Planck equation solved with generalized 
finite diffrences in 2D and 3D, Applied Mathematics and Computation, $368,124801,(2020)$. 

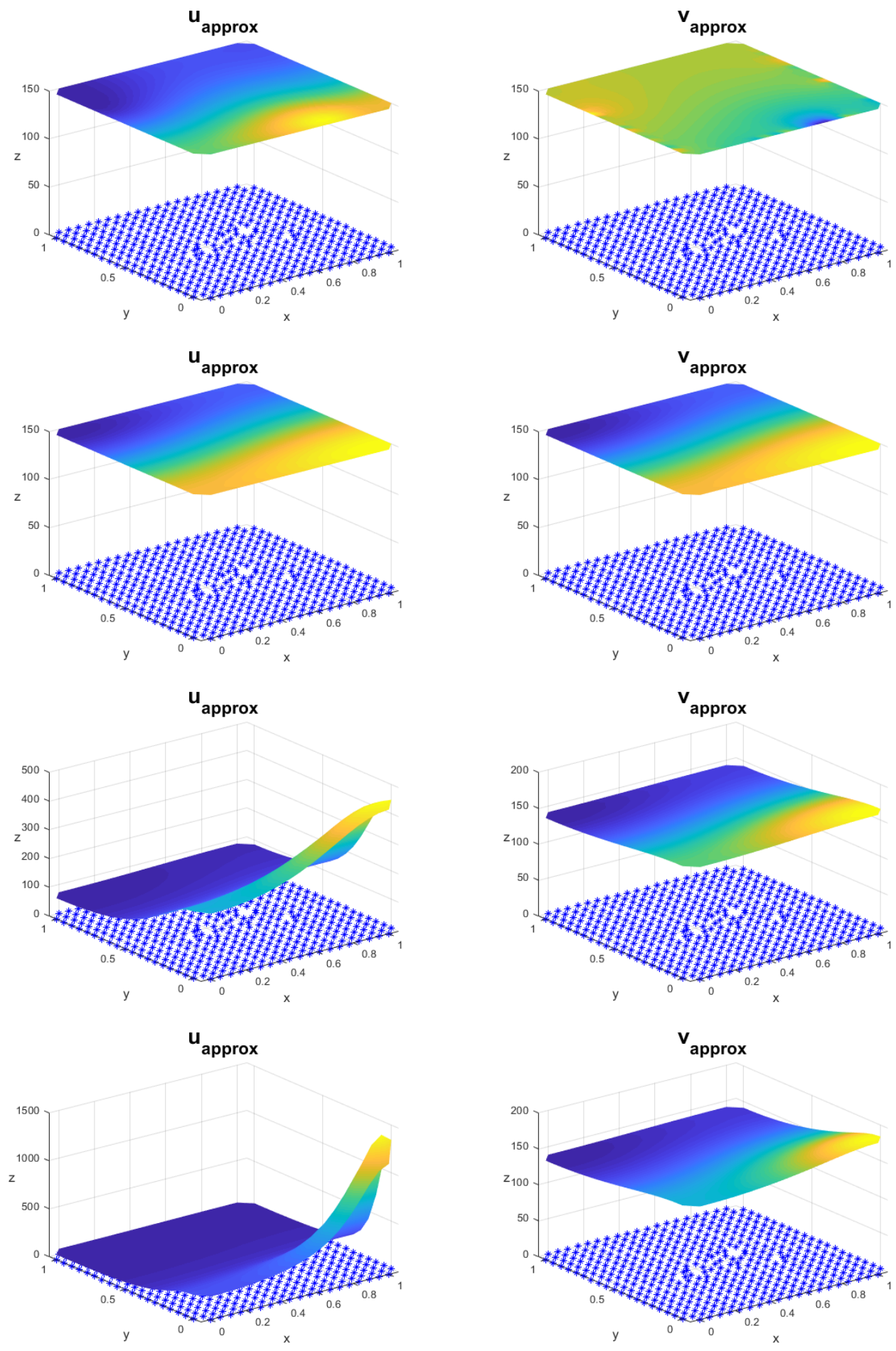

22

Figure 2: $u, v$-solution for $0.3,1,1.6$ and 1.625 seconds in the Example 1. 

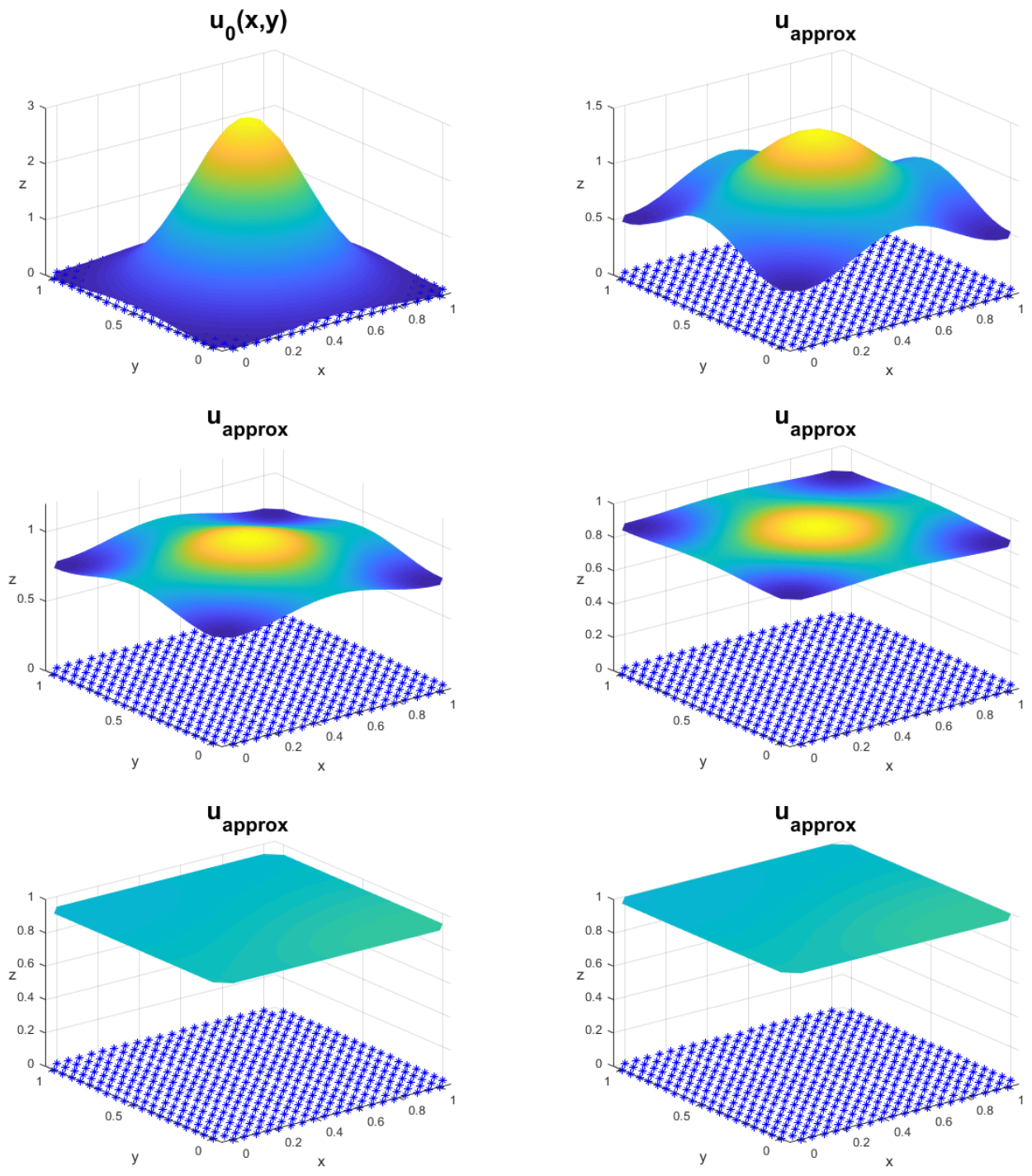

Figure 3: $u$-solution for $0,0.03,0.06,0.1,1$ and 6 seconds in the Example 2. 

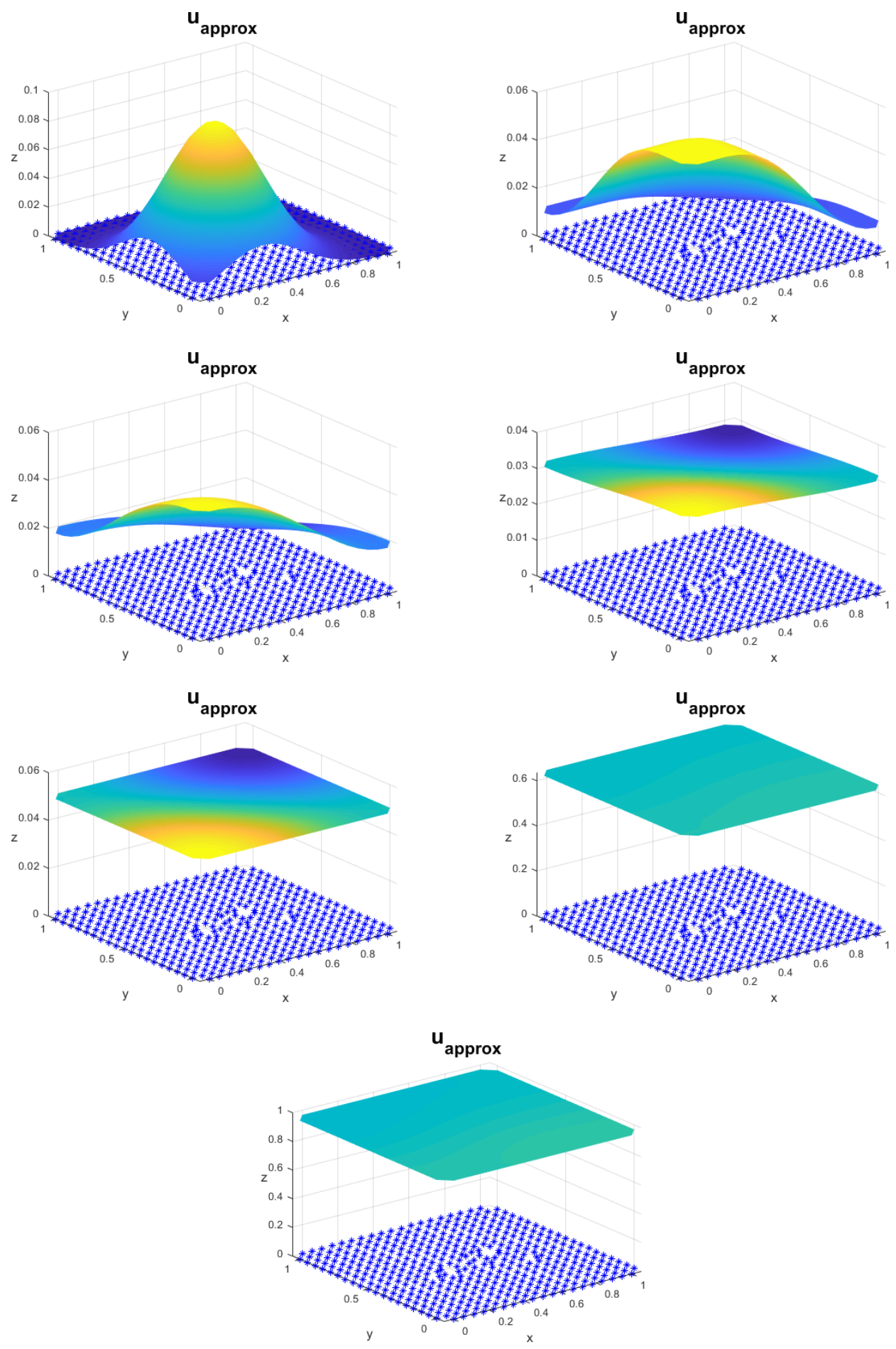

Figure 4: $u$-solution for 0, 0.03, 0.06, 0234 1, 6 and 10 seconds in the Example 3. 

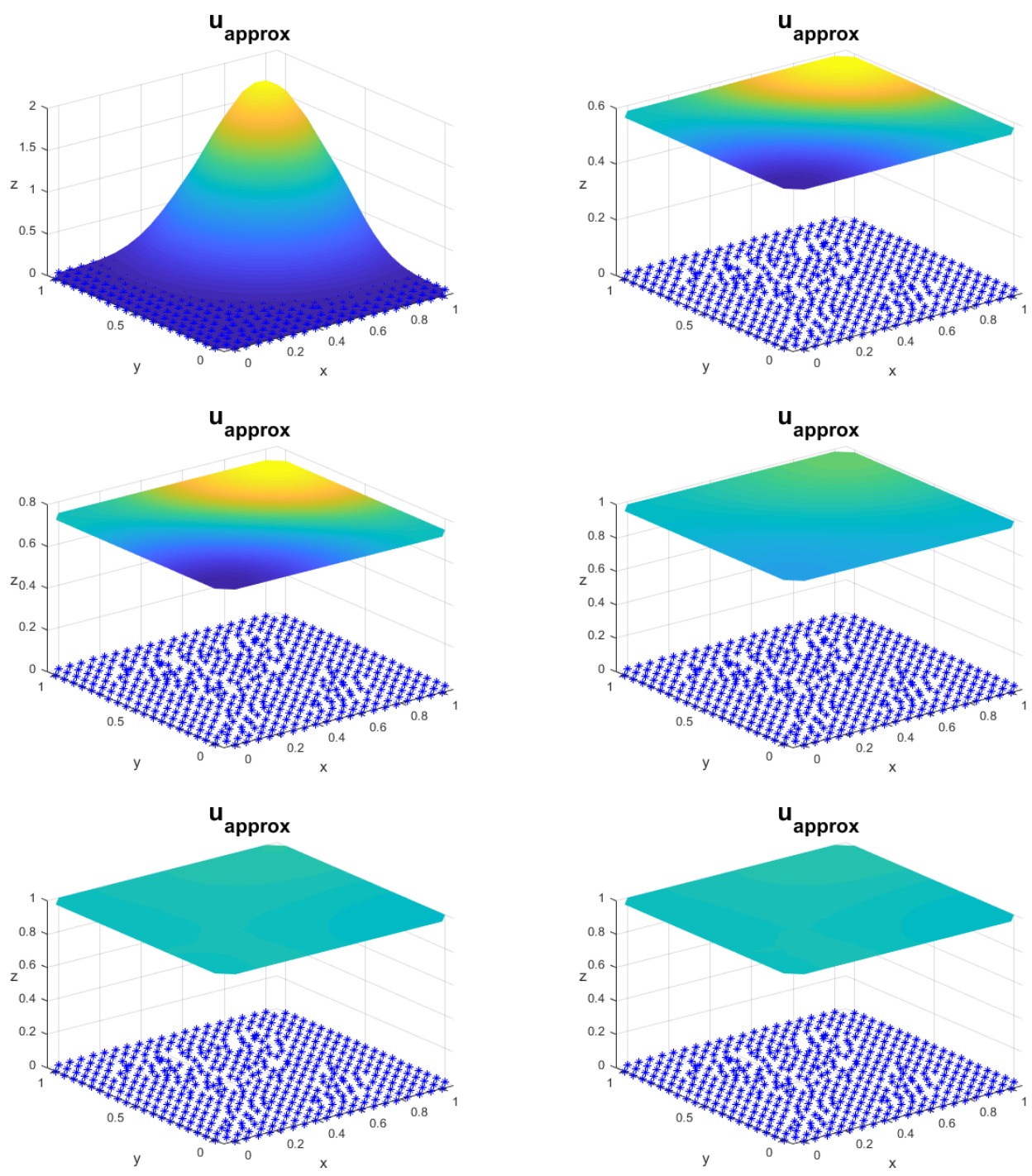

Figure 5: $u$-solution for 0, 0.5, 1, 3, 6 and 10 seconds in the Example 4. 


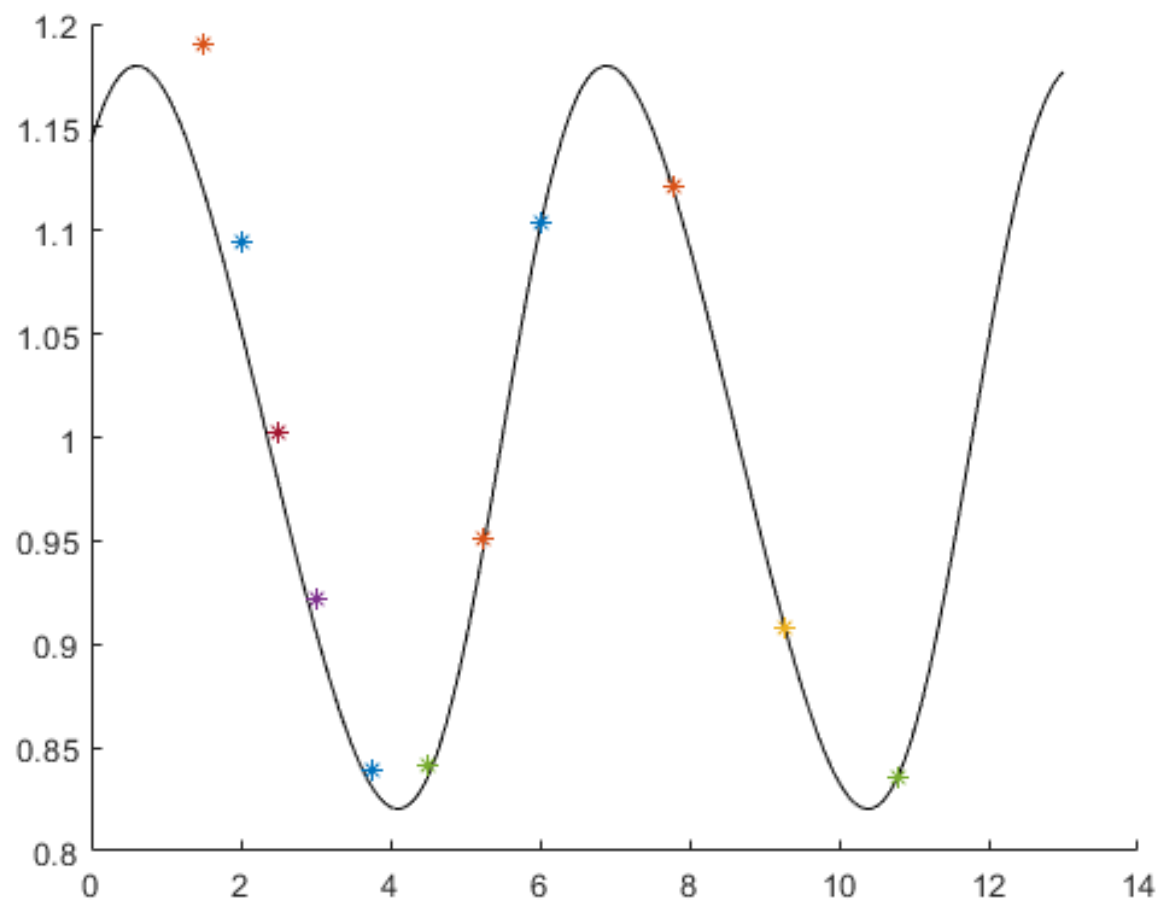

Figure 6: The solid line corresponds to the graphic of the function $L(t)$, the stars to the most distant value of the approximate solution $u$ at such time in Example 5. 


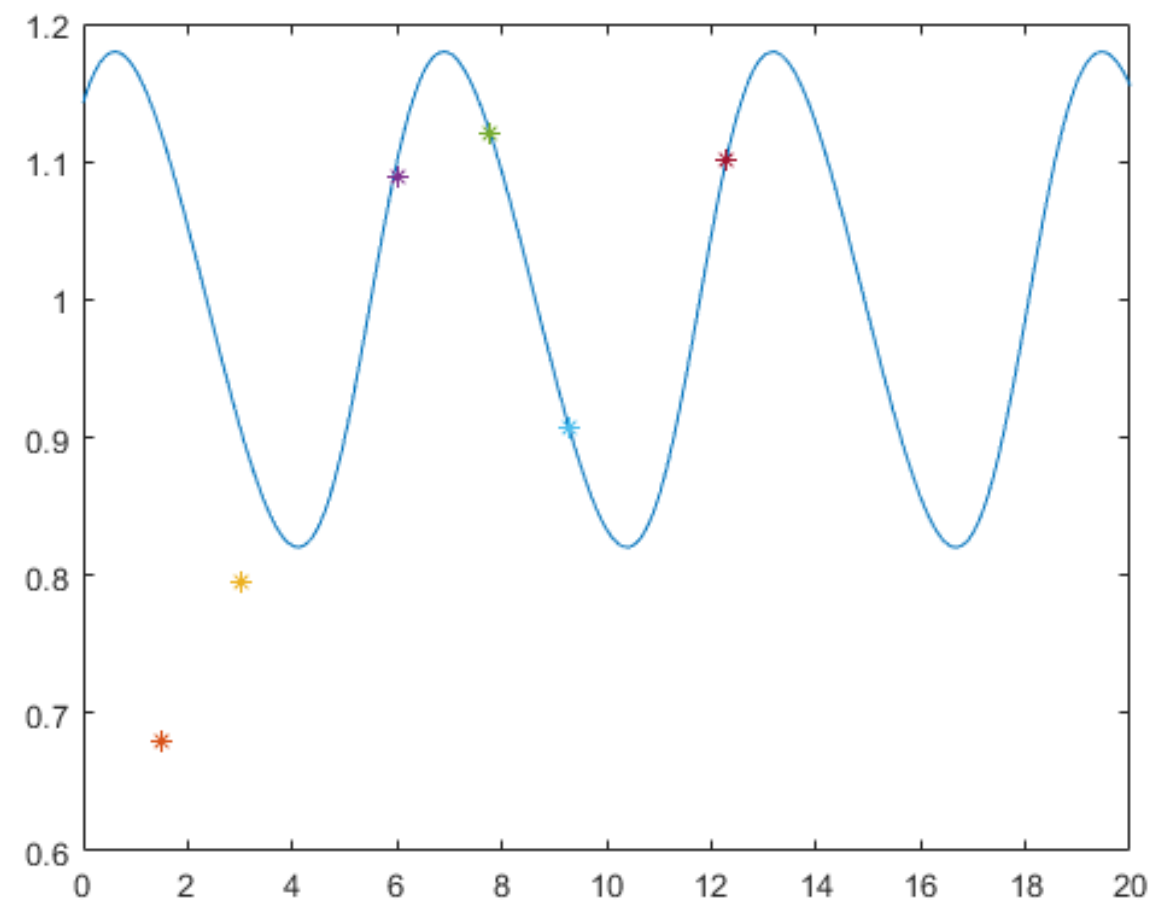

Figure 7: The solid line corresponds to the graphic of the function $L$, the stars to the most distant value of the approximate solution $u$ at such time in Example 6. 